\title{
1 Trans-segmental imaging in the spinal cord of behaving mice
}

2 Pavel Shekhtmeyster ${ }^{1,2, \#, ~ D a n i e l a ~ D u a r t e ~}{ }^{1, \#}$, Erin M. Carey ${ }^{1}$, Alexander Ngo ${ }^{1}$, Grace Gao ${ }^{1}$,

3 Jack A. Olmstead ${ }^{1,4}$, Nicholas A. Nelson ${ }^{1,3}$, and Axel Nimmerjahn ${ }^{1,{ }^{*}}$

$4 \quad{ }^{1}$ Waitt Advanced Biophotonics Center, The Salk Institute for Biological Studies, La Jolla, CA

5 92037, USA; ${ }^{2}$ Electrical and Computer Engineering Graduate Program, University of California,

6 San Diego, La Jolla, CA 92037, USA; ${ }^{3}$ Biological Sciences Graduate Program, University of

7 California, San Diego, La Jolla, CA 92037, USA; ${ }^{4}$ Neurosciences Graduate Program, University

8 of California, San Diego, La Jolla, CA 92037, USA

$9 \quad$ \#These authors contributed equally to this work.

10 *Correspondence: animmerj@salk.edu (A.N.)

\section{Abstract}

12 Spinal cord circuits play crucial roles in transmitting and gating cutaneous somatosensory

13 modalities, such as pain, but the underlying activity patterns within and across spinal segments

14 in behaving mice have remained elusive. To enable such measurements, we developed a

15 wearable widefield macroscope with a $7.9 \mathrm{~mm}^{2}$ field of view, subcellular lateral resolution, 2.7

$16 \mathrm{~mm}$ working distance, and $<10 \mathrm{~g}$ overall weight. We show that highly localized painful

17 mechanical stimuli evoke widespread, coordinated astrocyte excitation across multiple spinal

18 segments. 


\section{Introduction}

22 Spinal cord circuits are indispensable for sensory information processing and controlling

23 reflexive and adaptive behaviors, such as those driven by pain and itch, as demonstrated by

24 genetic, anatomical, electrophysiological, pharmacologic, and behavioral methods ${ }^{1}$. However,

25 these approaches lack the spatiotemporal resolution, resilience against tissue movement, or

26 cell-type specificity necessary to interrogate genetically defined cell types' activity patterns in

27 behaving animals. In vivo fluorescence imaging overcomes many of these barriers by permitting

28 high-speed, stable measurement of neuronal and non-neuronal activity in the spinal cord ${ }^{2}$.

Sensory stimuli can recruit cellular networks distributed across multiple spinal segments ${ }^{2,3}$ (each

$\sim 1-1.5 \mathrm{~mm}$ rostra-caudal length in mice $\left.{ }^{4}\right)$. Current fluorescence microscopes' optical properties

32 hamper the high-speed measurement of such distributed activity. While cellular-resolution

33 devices typically offer small fields of view (FOVs) covering less than one vertebra, large-FOV

34 microscopes ("macroscopes") provide limited spatiotemporal resolution, sensitivity, and

35 contrast, or are incompatible with use in freely moving mice ${ }^{2,5,6}$. Wearable widefield

36 macroscopes with millimeter-sized FOV have recently been developed ${ }^{7-9}$. Still, none offer the

37 cellular resolution, frame rates, or weights necessary for spinal cord imaging in mice. These

38 technical limitations hamper our ability to decipher how broadly dispersed cellular networks

39 contribute to somatosensory processing in behaving animals. Of particular interest, astrocytes

40 are emerging as essential regulators of pain signaling in the spinal dorsal horn ${ }^{10-13}$.

42 To address these challenges, we developed a wearable macroscope with custom-compound

43 microlenses that allows subcellular-resolution, high-speed activity measurements from

44 genetically defined cell types across multiple spinal segments $\left(7.9 \mathrm{~mm}^{2}\right.$ field of view) in

45 behaving animals. Using GFAP-GCaMP6f mice with calcium indicator expression in spinal 
astrocytes, we show that even highly localized noxious mechanical stimuli can evoke

47 widespread, coordinated calcium activity that spans multiple spinal segments. In contrast,

48 innocuous stimuli to the same cutaneous area led to only sparsely distributed calcium excitation,

49 suggesting categorical differences in these activity patterns' underlying cellular and molecular

50 mechanisms.

\section{Results}

53 A wearable widefield macroscope with custom-compound microlenses for millimeter-

54 scale, subcellular-resolution measurements in freely behaving mice. To meet the

55 application needs mentioned above, the design goals for our macroscope's optical system

56 included an $\sim 3.2 \times 2.5 \mathrm{~mm}$ FOV, high spatial resolution and contrast across the FOV $(\sim 3 \mu \mathrm{m})$, a

57 long working distance (> $2 \mathrm{~mm}$ ), and broad achromatic range (450-570 $\mathrm{nm}$ ). To provide

58 flexibility in the type and the number of light sources that can be coupled (e.g., DPSS lasers,

59 LEDs), a flexible PMMA fiber was chosen for excitation light delivery (Fig. 1a-b). Additional

60 design parameters included an $\sim 0.4$ numerical aperture, $~ 1.4 x$ magnification, and 36 mm track

61 length. To ensure that mice can comfortably wear the macroscope on its back, we aimed for an

62 overall weight of $<10 \mathrm{~g}$ (including all optics, optomechanical components, and electronics). To

63 minimize torque on the animal, we also opted for a folded imaging path (i.e., the short-pass

64 dichroic beamsplitter reflects fluorescence emission and transmits excitation light), thereby

65 lowering the integrated device's center of gravity.

66

67 Our optical design included eight custom microlenses from five different glass materials (Suppl.

68 Table 1). All glasses were high-index $(n=1.76-1.92)$ to increase optical power while

69 maintaining device compactness (Fig. 1a-b). Zemax modeling was used to optimize the lens

70 surface profile, radius of curvature, distance between surfaces, and glass types. We included a 
$71 \quad \# 0$ coverslip ( 100 $\mu$ m thickness) in our design to minimize optical aberrations when imaging

72 through a live animal-implanted glass window. Additionally, we accounted for optical filters

73 placed in the collimated space between the lenses required for fluorescence imaging. Following

74 optical modeling, the lenses and brass spacers were fabricated and assembled into two custom-

75 made barrel holders minimized in size and weight (Fig. 1a-b). Each assembled barrel weighed

$76<2.05 \mathrm{~g}$.

78 Next, we characterized our integrated system's optical performance by determining its limit of 79 resolution (LOR) across and point spread function (PSF) in the center of the FOV. For the LOR,

80 we measured $2.27 \mu \mathrm{m}$ in the center and halfway toward the edges of the FOV, and $2.85 \mu \mathrm{m}$ at

81 the FOV edges (Fig. 1C). The lateral and axial point spread function (PSF) of our integrated

82 system was $\sim 3.3 \mu \mathrm{m}$ and $\sim 17.7 \mu \mathrm{m}$, respectively, measured as full width half maximum (FWHM)

83 (Suppl. Fig. 1). Because the ability to retrieve image information can be limited by contrast,

84 independent of resolution, we quantified our macroscope's modulation transfer function (MTF).

85 Our integrated system's MTF10 (i.e., contrast at 10\%; Methods) was $169 \mathrm{lp} / \mathrm{mm}$ tangential

$86(2.96 \mu \mathrm{m}$ linewidth) and $193 \mathrm{lp} / \mathrm{mm}$ sagittal $(2.59 \mu \mathrm{m}$ linewidth) in the center of the FOV (Suppl.

87 Fig. 2). Notably, our macroscope performs well even at the FOV edges (Fig. 1f). The ability to

88 obtain high-contrast measurements becomes especially important in densely labeled tissue

89 (Figs. 1e-f, 2b, d).

91 Using a precision differential actuator and regularly spaced grid target with $500 \mu \mathrm{m}$ spacing, we

92 found that our wearable device had a $3.215 \times 2.465 \mathrm{~mm}$ FOV (Fig. 1d) and $2.727 \mathrm{~mm}$ working

93 distance (WD) (from the edge of the objective barrel to the microscopy target in air). This long

94 WD enables imaging through implantable micro-optics, such as microprisms (Suppl. Fig. 3).

95 Our fully assembled device measured $\sim 14 \times 26 \times 29 \mathrm{~mm}\left(4.423 \mathrm{~cm}^{3}\right.$ volume $)$ and weighed 9.8

96 g. Remarkably, 7-9 weeks-old mice could carry the device on their back without training. Open- 
97 field measurements confirmed that general locomotor activity (average running velocity, total

98 distance traveled, rearing activity) was largely unaffected (Suppl. Fig. 4).

99

100 Together, these data demonstrate that our miniature macroscope with custom-compound-

101 microlenses simultaneously provides an exceptional field of view, spatial resolution, contrast,

102 and working distance while retaining a small form factor and low weight suitable for spinal cord

103 imaging in freely moving mice.

104

105 High-speed trans-segmental imaging of sensory-evoked calcium activity in behaving

106 GFAP-GCaMP6f mice. To date, studies investigating somatosensory processing have been

107 limited to individual spinal segments (each $\sim 1-1.5 \mathrm{~mm}$ length in mice) or less, given previous

108 microscopes' technical limitations. As a result, little is known about the spatial extent and

109 temporal evolution of single-trial activity patterns across spinal segments.

111 To demonstrate the feasibility of high-speed, trans-segmental measurements with our wearable

112 macroscopes, we performed imaging in awake GFAP-GCaMP6f mice ( $\mathrm{N}=4)$ with constitutive

113 green-fluorescent calcium indicator GCaMP6f expression in astrocytes. Dorsal horn astrocytes

114 can exhibit noxious stimulus-evoked activity across large tissue regions (>500 $\mu \mathrm{m}$ rostrocaudal

115 extent, limited by FOV ${ }^{10,11}$, and this activity has been suggested to serve pain modulatory

116 functions ${ }^{11-13}$. However, its spatial extent and temporal dynamics across spinal segments remain

117 unknown. Determining the spatiotemporal properties of astrocytes' excitation is crucial to help

118 define its functional role (e.g., trans-segmental and -hemispheric sensitization), underlying

119 mechanisms (e.g., local or neuromodulatory signaling), and interventions to control it (e.g.,

120 through local or subpial viral vector injections) ${ }^{14}$. Using a rodent pincher system, we applied

121 quantifiable and repeatable pressure stimuli to the animal's proximal tail (duration: $1.5 \mathrm{~s} \pm 0.5 \mathrm{~s}$; 
pressure range: 50 - 1,100 g) (Methods) while imaging astrocytes' calcium excitation across L2locomotor activity, we placed the mice on a spherical treadmill during imaging. To quantify sensory-evoked astrocyte responses comprehensively, we tiled the FOV with equally sized ( 10 $\mu \mathrm{m} \times 10 \mu \mathrm{m})$ ROls, excluding blood vessel regions (Methods).

Innocuous tail pinch stimuli $(p<200 \mathrm{~g}$ ) led to only sparse, low amplitude transients (most $<20 \%$ $\Delta \mathrm{F} / \mathrm{F}$ ) along the length of the $>2,000 \mu \mathrm{m}$ laminectomy (Fig. 2b-c; Suppl. Movie 1). In contrast, noxious tail pinch ( $p>500 \mathrm{~g}$ ) evoked widespread coordinated calcium transients bilaterally and across spinal segments, with many transients exceeding $40 \% \Delta \mathrm{F} / \mathrm{F}$ (Fig. 2d-f; Suppl. Movie 2). While noxious stimulus application often evoked locomotor activity, running in the absence of tail pinch did not evoke comparable population activity (Fig. 2c, e; Suppl. Movie 1) (Methods), consistent with previous work ${ }^{10}$. Calcium transients evoked by noxious pinch had an onset latency of $2.04 \pm 0.07 \mathrm{~s}$ (population average) (Fig. $2 \mathrm{~g}$ ), amplitude of $0.41 \pm 0.01 \Delta \mathrm{F} / \mathrm{F}$ (individual ROIs) and $0.49 \pm 0.14 \Delta \mathrm{F} / \mathrm{F}$ (population average) (Fig. 2h), and duration of $2.70 \pm 0.01 \mathrm{~s}$

137 (individual ROIs) and $2.88 \pm 0.97 \mathrm{~s}$ (population average) (Fig. 2i). The amplitude and duration of population transients were comparable on the left and right sides of the spinal cord (Fig. 2j, k) (Methods). Based on onset latency, the activity originated in central gray matter regions of each hemisphere (Fig. 2e). Together, these data demonstrate that our wearable macroscopes are capable of high-resolution, high-speed bilateral and trans-segmental measurement of sensoryevoked activity in genetically defined cells of behaving mice.

\section{Discussion}

145 The technical capability of our wearable macroscopes for millimeter-scale, subcellular-resolution 146 measurements was enabled by custom-compound microlenses (Fig. 1a, c-d, f). These custom 
147 optics have a theoretical lateral and axial PSF of $0.55 \mu \mathrm{m}$ and $9.03 \mu \mathrm{m}$, respectively, based on

148 Zemax calculations. Given the experimentally measured lateral and axial resolution of $\sim 2.3-3.3$

$149 \mu \mathrm{m}$ and $17.7 \mu \mathrm{m}$, respectively, this suggests that our wearable macroscopes are currently

150 limited by the image sensor's resolution ( $2.63 \mu \mathrm{m}$ in the object space). Therefore, future CMOS

151 sensor upgrades are expected to improve the device's overall resolution even further. Our

152 device's modular design greatly facilitates such upgrades (Methods).

154 Fig. 2 demonstrates our wearable macroscopes' ability to perform high-speed, high-sensitivity

155 functional measurements from genetically defined cells across multiple spinal segments. The

156 properties of tail pinch- and running-evoked calcium transients in GFAP-GCaMP6f mice (e.g.,

157 onset latency, duration) were consistent with previous studies ${ }^{2,10}$. Notably, the significantly

158 larger FOV allowed us to reveal that even focally delivered sensory stimuli could evoke

159 remarkably widespread, trans-segmental calcium excitation in dorsal horn astrocytes, while

160 isolated running bouts did not. Future studies will need to determine the full extent (in

161 rostrocaudal and dorsoventral directions), underlying mechanisms (e.g., descending

162 noradrenergic, local glutamatergic signaling, gap junction involvement), behavioral

163 dependencies (e.g., motor activity), and modulatory effects of this widespread astrocyte activity

164 on spinal neural circuits and behavior (e.g., trans-segmental sensitization $)^{14-16}$. Innocuous pinch

165 evoked only sparse activity in isolated ROI clusters (Fig. 2b). The location of these clusters may

166 provide clues about regional differences in spinal sensory processing and serves as a reminder

167 that data from small FOVs can introduce sampling bias.

169 In summary, the technical capabilities, small form factor, and low weight of our wearable

170 macroscopes pave the way for a wide range of exciting neuroscience applications. The

171 repeatable and quantifiable stimuli employed in our study should facilitate comparative

172 measurements in other transgenic mouse lines, such as recordings in genetically defined 
173 neuronal subpopulations to reveal their activity patterns in relation to astrocytes. Simultaneous

174 measurement of multiple cell types' activity patterns should be feasible given our macroscopes'

175 450-570 $\mathrm{nm}$ achromatic range but will require custom multiband filters optimized for large

176 incidence angles (Methods). By allowing measurements from large tissue areas and distributed

177 cell networks in behaving animals, our macroscopes may find novel applications in various

178 disease settings (e.g., structural/functional regeneration after spinal cord injury ${ }^{17}$ ) and larger

179 experimental models (e.g., transgenic rats $^{7}$, marmoset ${ }^{18}$ ). Our macroscope's long working

180 distance combined with implantable optics, such as microprisms (Suppl. Fig. 3), should enable

181 high-speed translaminar measurement of sensorimotor activity from and across spinal laminae

182 (e.g., related to motor control). Imaging during standard mouse behavioral assays should be

183 feasible given the minimal mobility impediment mice showed during the open field test (Suppl.

184 Fig. 4). Altogether, we expect end-users of our macroscopes to achieve real-time, high-

185 resolution visualization of cellular activity patterns across large FOVs in a multitude of

186 behavioral and disease contexts. 


\section{References}

1901 Koch, S. C., Acton, D. \& Goulding, M. Spinal Circuits for Touch, Pain, and Itch. Annu

191 Rev Physiol 80, 189-217, doi:10.1146/annurev-physiol-022516-034303 (2018).

1922 Nelson, N. A., Wang, X., Cook, D., Carey, E. M. \& Nimmerjahn, A. Imaging spinal cord 193 activity in behaving animals. Exp Neurol 320, 112974,

194 doi:10.1016/j.expneurol.2019.112974 (2019).

1953 Abraira, V. E. \& Ginty, D. D. The sensory neurons of touch. Neuron 79, 618-639, 196 doi:10.1016/j.neuron.2013.07.051 (2013).

1974 Harrison, M. et al. Vertebral landmarks for the identification of spinal cord segments in 198 the mouse. Neuroimage 68, 22-29, doi:10.1016/j.neuroimage.2012.11.048 (2013).

1995 Aharoni, D. \& Hoogland, T. M. Circuit investigations with open-source miniaturized

200 microscopes: Past, present and future. Front Cell Neurosci 13, 141, 201 doi:10.3389/fncel.2019.00141 (2019).

2026 Chen, S. et al. Miniature fluorescence microscopy for imaging brain activity in freely203 behaving animals. Neurosci Bull 36, 1182-1190, doi:10.1007/s12264-020-00561-z 204 (2020).

2057 Scott, B. B. et al. Imaging Cortical Dynamics in GCaMP Transgenic Rats with a Head206 Mounted Widefield Macroscope. Neuron 100, 1045-1058 e1045, 207 doi:10.1016/j.neuron.2018.09.050 (2018).

2088 Rynes, M. L. et al. Miniaturized head-mounted microscope for whole-cortex mesoscale 209 imaging in freely behaving mice. Nat Methods 18, 417-425, doi:10.1038/s41592-021$210 \quad$ 01104-8 (2021).

2119 Guo, C. et al. Miniscope-LFOV: A large field of view, single cell resolution, miniature 212 microscope for wired and wire-free imaging of neural dynamics in freely behaving animals. bioRxiv, doi: https://doi.org/10.1101/2021.1111.1121.469394 (2021). 
21410 Sekiguchi, K. J. et al. Imaging large-scale cellular activity in spinal cord of freely 215 behaving mice. Nat Commun 7, 11450, doi:10.1038/ncomms11450 (2016).

21611 Kohro, Y. et al. Spinal astrocytes in superficial laminae gate brainstem descending 217 control of mechanosensory hypersensitivity. Nat Neurosci 23, 1376-1387, 218 doi:10.1038/s41593-020-00713-4 (2020).

$21912 \mathrm{Xu}, \mathrm{Q}$. et al. Astrocytes contribute to pain gating in the spinal cord. Sci Adv 7, eabi6287, 220 doi:10.1126/sciadv.abi6287 (2021).

$221 \quad 13 \quad$ Nam, Y. et al. Reversible Induction of Pain Hypersensitivity following Optogenetic 222 Stimulation of Spinal Astrocytes. Cell Rep 17, 3049-3061, 223 doi:10.1016/j.celrep.2016.11.043 (2016).

22414 Merten, K., Folk, R. W., Duarte, D. \& Nimmerjahn, A. Astrocytes encode complex 225 behaviorally relevant information. bioRxiv, doi:

$226 \quad$ https://doi.org/10.1101/2021.1110.1109.463784 (2021).

22715 Orts-Del'Immagine, A., Dhanasekar, M., Lejeune, F. X., Roussel, J. \& Wyart, C. A 228 norepinephrine-dependent glial calcium wave travels in the spinal cord upon acoustovestibular stimuli. Glia, doi:10.1002/glia.24118 (2021).

$23016 \quad$ Mu, Y. et al. Glia Accumulate Evidence that Actions Are Futile and Suppress Unsuccessful Behavior. Cell 178, 27-43 e19, doi:10.1016/j.cell.2019.05.050 (2019).

23217 Ceto, S., Sekiguchi, K. J., Takashima, Y., Nimmerjahn, A. \& Tuszynski, M. H. Neural Stem Cell Grafts Form Extensive Synaptic Networks that Integrate with Host Circuits after Spinal Cord Injury. Cell Stem Cell 27, 430-440 e435,

23618 Kondo, T. et al. Calcium Transient Dynamics of Neural Ensembles in the Primary Motor 237 Cortex of Naturally Behaving Monkeys. Cell Rep 24, 2191-2195 e2194, 238 doi:10.1016/j.celrep.2018.07.057 (2018). 
a

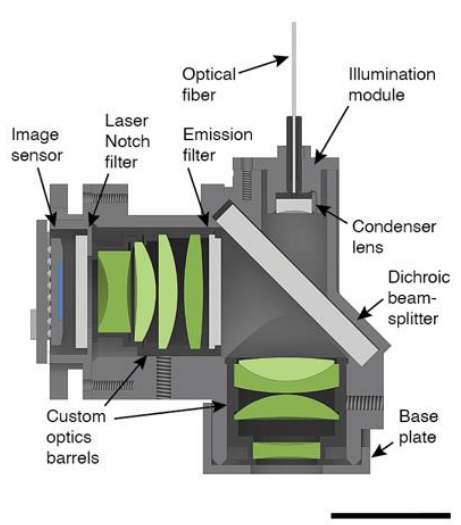

C
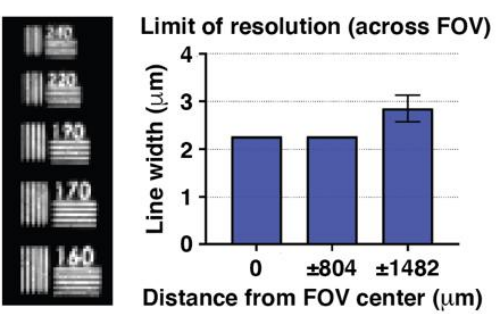

b

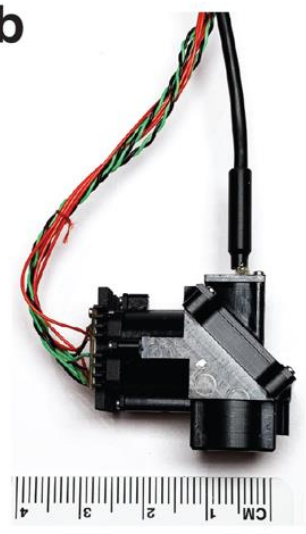

d
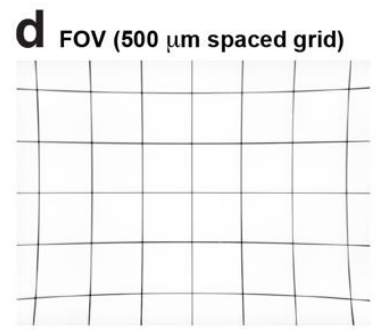

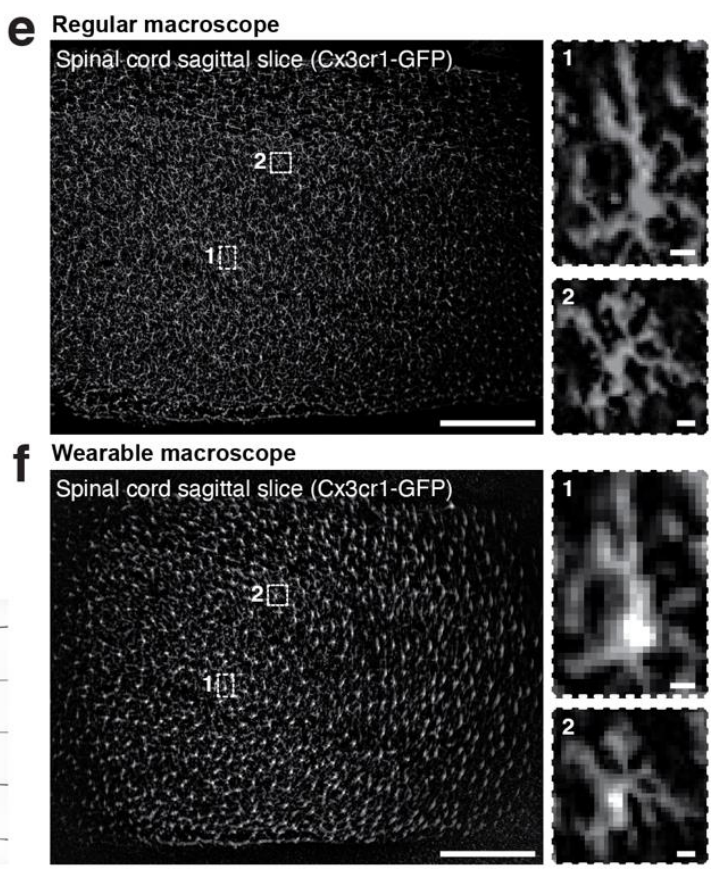

Fig. 1 | Wearable macroscopes with custom-compound-microlenses for millimeter-scale,

subcellular-resolution measurements in freely behaving mice. a, Cross-section of the

wearable macroscope and its custom compound micro-optics (green). Scale bar, $10 \mathrm{~mm} . \mathbf{b}$,

Photo of the fabricated device. c, Limit of resolution across the FOV. Displayed values are

averages across the horizontal and vertical line target results from comparable positions to the

left and right of the FOV center. Spatial frequencies were converted to line widths. The data are

presented as mean \pm s.e.m. The error bars at $0 \mu \mathrm{m}$ and $\pm 804 \mu \mathrm{m}$ are too small to be visible. $\mathbf{d}$,

249 Image of a 500- $\mu \mathrm{m}$ spacing grid target acquired with the integrated device demonstrating its

$250 \sim 3.2 \times 2.5 \mathrm{~mm}$ FOV. e, Left, fluorescence image of a $20-\mu \mathrm{m}$-thick sagittal spinal cord section

251 from a Cx3cr1 $1^{+/ G F P}$ mouse with labeled microglia acquired with a commercial benchtop

252 macroscope. Right, blow-up of the two subregions indicated on the left showing individual

253 microglial cells. f, Left, fluorescence image of the same spinal cord section as in c acquired with

254 the wearable macroscope. Right, blow-up of the subregions indicated on the left. Over 1,000

255 individual microglial cells are visible. Scale bars in e and f, $500 \mu \mathrm{m}$ (left) and $10 \mu \mathrm{m}$ (right). 
bioRxiv preprint doi: https://doi.org/10.1101/2021.12.23.474042; this version posted December 24,2021 . The copyright holder for this preprint (which was not certified by peer review) is the author/funder, who has granted bioRxiv a license to display the preprint in perpetuity. It is made available under aCC-BY-NC-ND 4.0 International license.

a
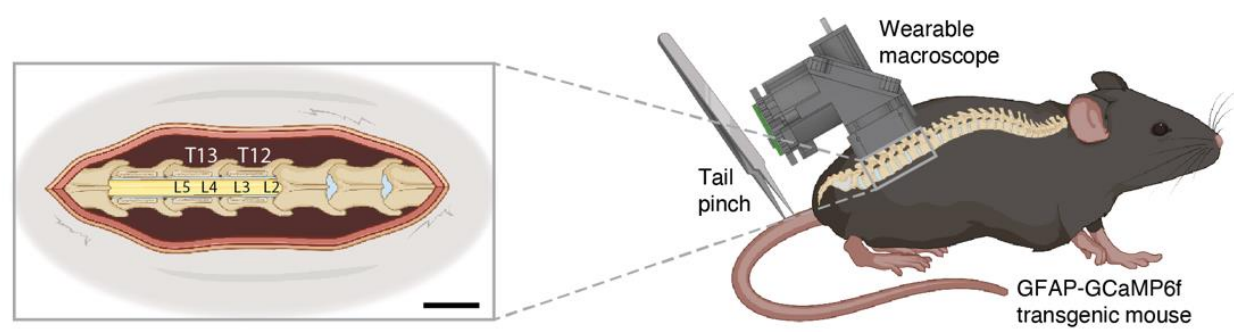

b

Innocuous pinch trial

$\square=20 \% \Delta \mathrm{F} / \mathrm{F}$

$\square=40 \% \Delta \mathrm{F} / \mathrm{F}$
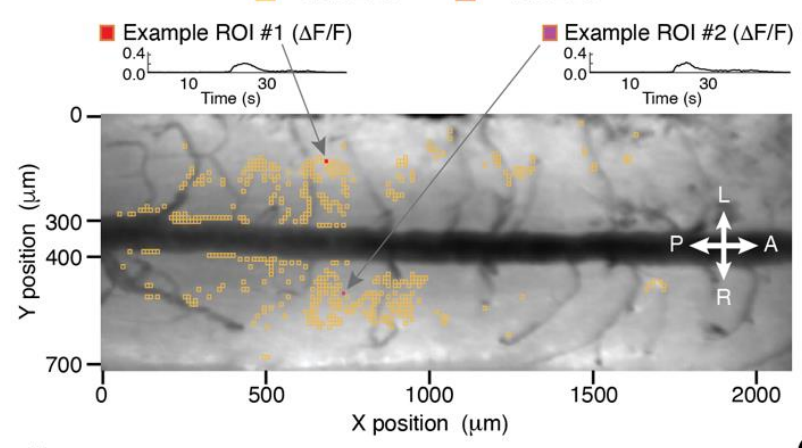

C

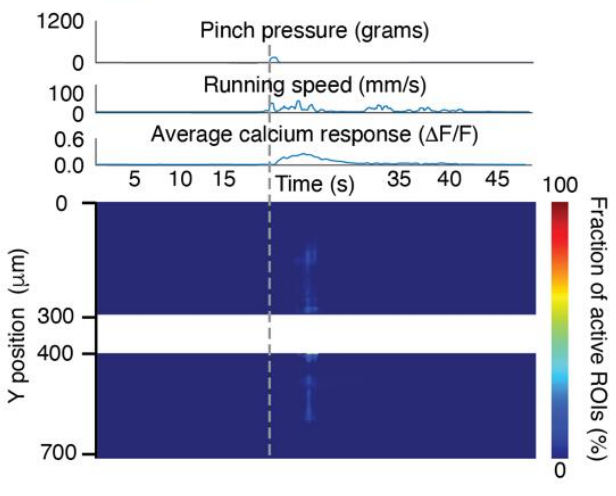

d

Noxious pinch trial

$\square=20 \% \Delta \mathrm{F} / \mathrm{F} \quad \square=40 \% \Delta \mathrm{F} / \mathrm{F}$

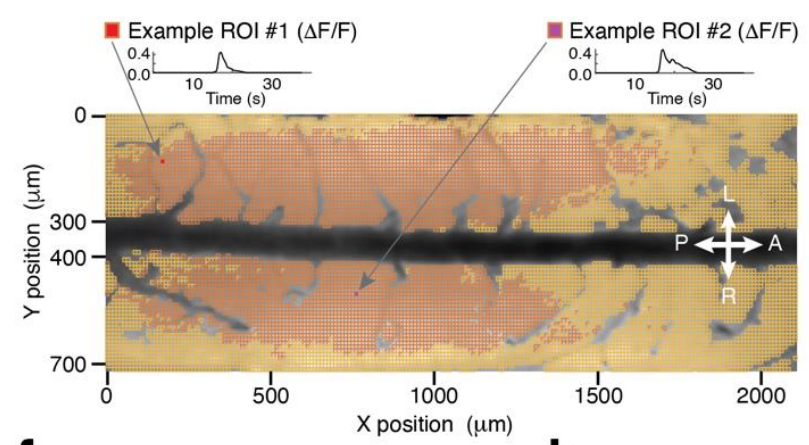

e
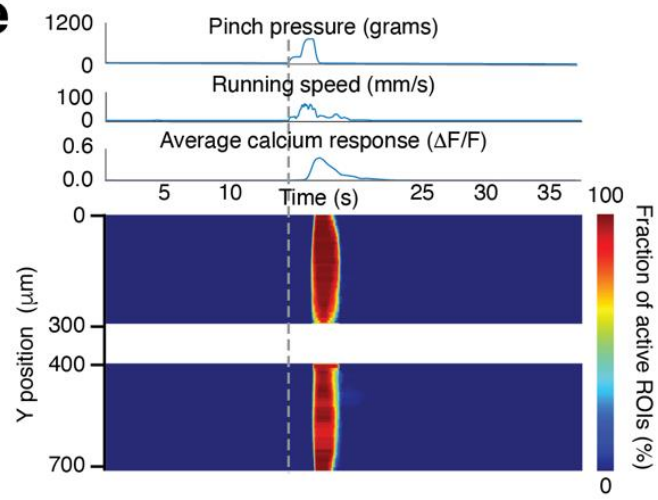

f

h Amplitude of astrocyte calcium transients

Amplitude of astrocyte calcium transients

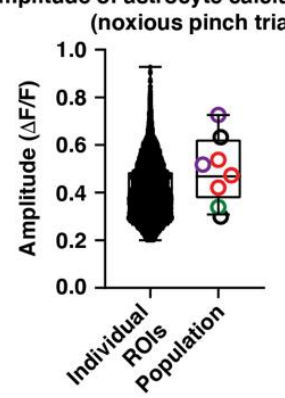

O Mouse 1

- Mouse 2

- Mouse 3

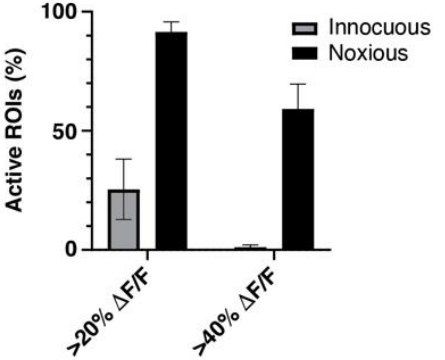

9 Onset latency of astrocyte calcium transients I

Duration of astrocyte calcium transients
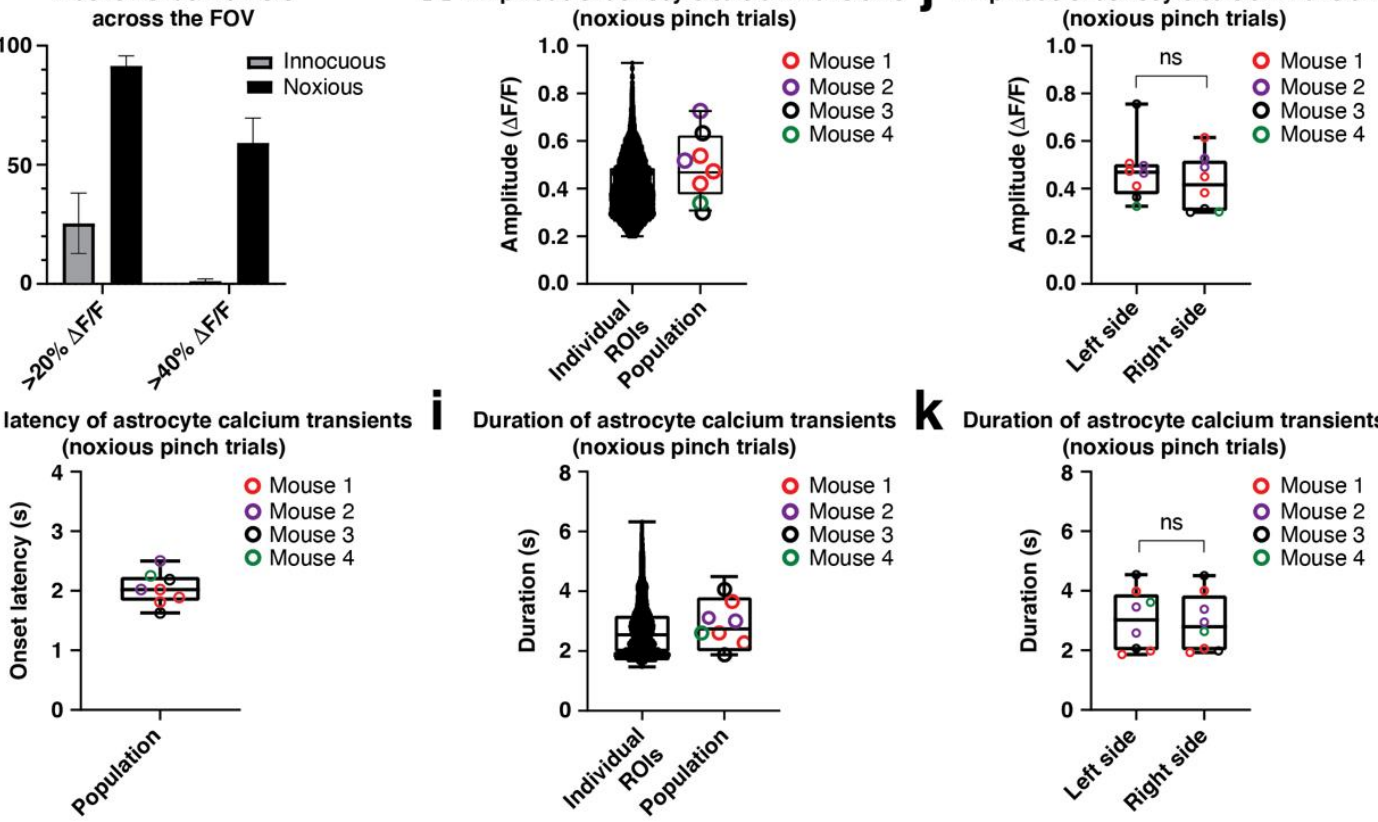

Duration of astrocyte calcium transients

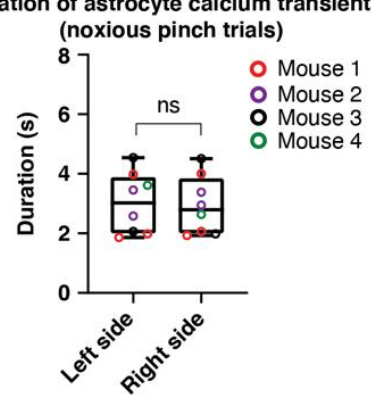


Fig. 2 | High-speed trans-segmental imaging of sensory-evoked calcium activity in behaving GFAP-GCaMP6f mice. a, Schematic of the experimental approach. A laminectomy was performed over the lumbar spinal cord of GFAP-GCaMP6f mice $(\mathrm{N}=4)$. The wearable macroscope was mounted above it. Pinch stimuli of different intensities and set duration (1.5 $\mathrm{s} \pm$ $0.5 \mathrm{~s})$ were delivered to the awake animal's proximal tail. Running speed was recorded by placing the animal on a spherical treadmill (Methods). Left inset scale bar, 1,400 $\mu \mathrm{m}$. b, Average intensity projection image from a time-lapse recording taken at $\sim 50 \mu \mathrm{m}$ focal depth below the pia overlaid with $10 \mu \mathrm{m} \times 10 \mu \mathrm{m}$ ROls. Only ROls with at least $20 \%$ (yellow) and $40 \% \Delta \mathrm{F} / \mathrm{F}$ (orange) in response to an innocuous pinch $(\mathrm{p}<200 \mathrm{~g})$ are shown. Innocuous stimuli evoked only sparse astrocyte activity on the left $(L)$ and right $(R)$ sides of the spinal cord and across anterior $(A)$ and posterior $(P)$ lumbar spinal segments (Suppl. Movie 1). Two example ROl's calcium transients are shown above the activity map. c, Innocuous tail pinch-evoked activity for the example recording shown in b. Each row depicts the percent of active ROIs (>20\% $\Delta \mathrm{F} / \mathrm{F}$ ) across a given mediolateral $(\mathrm{Y})$ position. The corresponding pressure stimulus,

271 locomotor activity, and average calcium transient across the FOV are shown above the activity

272 heat map. d, Activity map from the same animal and focal depth as in b for a noxious pinch trial

273 ( $p>500 \mathrm{~g})$. Noxious stimuli evoked widespread, bi-lateral astrocyte excitation across spinal

274 segments (Suppl. Movie 2). Two example ROl's calcium transients are shown above the

275 activity map. e, Noxious tail pinch-evoked astrocyte activity across different $Y$ positions.

276 Pressure stimulus amplitude, locomotor activity, and average calcium signal across the FOV are

277 shown above the activity heat map. f, Population data showing the percent of active ROls with

278 at least $20 \%$ or $40 \% \Delta F / F$ for innocuous and noxious stimulus trials. $\mathbf{g}$, Population data showing

279 the average calcium transient onset latency for noxious pinch trials. $\mathbf{h}-\mathbf{i}$, Population data

280 showing the individual ROI and average calcium response amplitude (h) and duration (i) for

281 noxious pinch trials. j-k, Population data showing the average calcium transient amplitude (j)

282 and duration $(k)$ on the left and right sides of the spinal cord for noxious pinch trials. The data in 
$283 \mathrm{f}$ are from 9 innocuous and 8 noxious pinch trials in 4 mice. The data in $\mathrm{h}$ (left) are from 67,696

284 ROls with $\Delta F / F>20 \%, 8$ recordings, and 4 mice. The data in i (left) are from 62,330 ROls with

$285 \Delta \mathrm{F} / \mathrm{F}>40 \%, 8$ recordings, and 4 mice. The data in $\mathrm{g}, \mathrm{h}-\mathrm{i}$ (right), and $\mathrm{j}-\mathrm{k}$ are from 8 recordings

286 and 4 mice. Paired t-tests determined $P$ values, and all bar plots are presented as mean \pm

287 s.e.m. The box and violin plots mark the median and the 25th and 75th percentiles, and the

288 whiskers cover the minimum and maximum of the data. 


\section{Methods}

292 Miniature macroscope design. Our wearable macroscope includes six major components: the

293 custom optics barrels (including eight microlenses), fluorescence filters, main body, image

294 sensor module, illumination module, and base plate (Fig. 1a).

Optical design. The optical design was generated de novo using Zemax optical modeling software (Zemax LLC) and manual calculations. The final design form resembled a double

298 Petzval-type short-conjugate relay. This fairly unique configuration allows for simultaneously

299 large numerical aperture (NA) and field of view (FOV) while maintaining a reasonable principal 300 ray angle ( $<15$-degree half-cone incident on the dichroic and emission filters). Each half of the 301 optical system included a negative element, fitting the Petzval-type description, which acts both 302 as a field-flattener and to shorten the physical length of the optical system, akin to a telephoto 303 configuration. During the design phase, geometric distortion was deliberately allowed to

304 increase to $8.1 \%$ to gain improvement across the other Seidel aberrations (spherical, coma, 305 astigmatism, and field curvature). The final optical design, which involved extensive global 306 optimization, utilized eight lens elements made from five different glass materials (Suppl. Table

307 1) housed in two separate custom mechanical housings/barrels for easy assembly (Optics

308 Technology Inc.). Both halves of the optical train were optimized together rather than

309 independently, with each half compensating for the aberrations of the other to ensure high

310 performance. To achieve near-uniform optical performance across the FOV, we incorporated a

311 small field aperture at the plane of the primary emission filter, which allowed for an $~ 37 \%$

312 improvement in resolution at the FOV edges while reducing light collection by only $\sim 11 \%$ (based

313 on Zemax calculations). Light collection and resolution in the central FOV ( 2 $\mathrm{mm}$ diameter)

314 were unaffected by this aperture. Based on Zemax calculation, the numerical aperture (NA) of 315 our wearable macroscopes is 0.39 . 
317 Our macroscope uses a short-pass dichroic beamsplitter (FF496; Semrock Inc) and primary

318 (ET525/50m; Chroma Technology Corp.) and secondary emission filters (FF01-496/LP;

319 Semrock Inc.). These filters were chosen to maximize the emission signal from the green

320 fluorescent genetically encoded calcium indicator GCaMP, currently the most advanced and

321 common calcium reporter in use. No excitation filter was necessary because we employed a

$322473 \mathrm{~nm}$ DPSS laser as the excitation light source (MBL-FN-473; CNI Optoelectronics Tech.

323 Co.).

325 Standard interference-type filters are designed for a particular angle of incidence (45 degrees

326 for dichroic beamsplitters and 0 degrees for emission filters), with a tolerance of around $\pm 5-7$

327 degrees. To achieve a large FOV while maintaining reasonable device dimensions, we

328 deliberately allowed a larger than usual principal ray incidence angle on the filters $( \pm 15$

329 degrees). Increasing the incidence angle widens the passband of interference filters while

330 shifting it toward shorter wavelengths. In particular, for the 45-degree dichroic beamsplitter,

331 placed in the center of the optical train, light on the side closer to the image sensor reflects at a

332 significantly larger angle than on the opposite side (60- and 30-degree incidence angle,

333 respectively). This feature results in the dichroic beamsplitter's spectral edge shifting by as

334 much as $\pm 25 \mathrm{~nm}$ around its nominal edge of $\lambda=491 \mathrm{~nm}$ (at 45-degree incidence). On the side

335 closer to the image sensor, the beamsplitter's spectral edge shifts to $\lambda=516 \mathrm{~nm}$, rejecting more

336 of the fluorescence signal, causing the corresponding image region to get darker. On the

337 opposite side, the spectral edge shifts to $\lambda=466 \mathrm{~nm}$, accepting more stray excitation light,

338 causing a brighter image region with lower contrast. For emission filters, the increased

339 incidence angle results in the acceptance of more stray excitation light. We solved the stray light

340 problem by placing a secondary emission filter in front of the image sensor, blocking most stray

341 excitation light. The primary and secondary emission filters are approximately located at

342 conjugate planes and, therefore, complement each other in angular performance. 
344 The excitation light was delivered to the macroscope using a flexible multi-mode fiber (MMF)

345 with a $200 \mu \mathrm{m}$ core and 0.22 NA (FG200UEA; Thorlabs). To reduce laser speckle, we employed

346 a square-core fiber (FP150QMT; Thorlabs) and a device that generates time-dependent mode-

347 mixing within the MMF on a fast time scale through induced fiber bending (quick relative to the

348 image acquisition speed). A square-core fiber was chosen because of its high degree of

349 inherent mode-mixing and near isotropic mixing of modes over a large range of fiber-bend radii.

350 A precision optical spacer and condenser lens (part no. 84-377; Edmund Optics) were used to

351 collimate the illumination light. Note that optical fibers were chosen because they provide

352 flexibility in coupling different light sources (e.g., DPSS lasers and LEDs), providing unique

353 opportunities for future all-optical interrogations of cellular activity in behaving animals.

Optomechanical design. The macroscope housing was designed using CAD software (Autodesk

356 Inventor) and included four modules (main body, sensor mount, illumination module, and base

357 plate). The main body houses the imaging optics and fluorescence filters. The illumination

358 module delivers the excitation light. The image sensor module mounts and provides heat

359 dissipation for the CMOS sensor. The detachable base plate allows reproducible mounting,

360 facilitating chronic imaging experiments. This modular design allows for modification of

361 individual components without affecting the rest of the device (e.g., to upgrade the image

362 sensor, incorporate an integrated LED instead of a fiber-coupled laser, or modify the baseplate

363 to pursue an alternative mounting strategy).

365 The individual lens elements and primary emission filter were assembled inside two barrels to 366 ensure light tightness. All fluorescence filters were edge-blackened (Sharpie Paint Pen). The 367 dichroic cover, which also mounts the illumination module, was coated with highly absorptive 368 black paint (Avian Black-S) to reduce reflections of back-scattered stray light. 
370 Most of the housing was fabricated from light-weight black polyether ether ketone (PEEK), a

371 thermoplastic opaque to visible and infrared (IR) light (near-IR light with an emission peak of

372 around $850 \mathrm{~nm}$ was used to illuminate the behavioral setup during video recordings). The

373 sensor mount was machined from aluminum to help dissipate heat generated by the image

374 sensor, thereby reducing sensor noise. The housing was optimized over several iterations such

375 that the fully assembled device weighed $<10 \mathrm{~g}$, ensuring that the animals could comfortably

376 carry the macroscope on their back. We employed a folded imaging path to lower the device's

377 center of gravity and minimize torque on the animal (i.e., the short-pass dichroic beamsplitter

378 reflected fluorescence emission and transmitted excitation light). Five-axis computer numerical

379 control (CNC) milling was selected for ease of fabrication. This technique influenced the design

380 process as the complex geometry needed to be confined to dimensions accessible to the CNC

381 mill.

382

383 Electronic design. Fluorescence emission was detected with a CMOS sensor

384 (MT9M024IX3STC, Aptina Imaging), which was chosen for its high-sensitivity (5.48 and 6.7

$385 \mathrm{~V} /$ lux-sec for the RGB and monochrome versions, respectively), resolution (1280 x 960 pixels;

$3863.75 \mu \mathrm{m}$ pixel spacing, corresponding to $2.63 \mu \mathrm{m}$ at the object plane), and frame rate (45 fps at

387 full resolution). Note that high frame rates are essential for spinal cord recordings given the

388 rapid, large-amplitude, and non-uniform tissue displacements in this region during animal

389 behavior $^{10}$.

390

391 While the image sensor can record data at 14-bit (linear gamma), all data were recorded at 12-

392 bit depth. The sensor was mounted on a custom miniature printed circuit board (PCB; Axelsys), 393 interfaced with an Aptina data-acquisition board (AGB1N0CS-GEVK), and controlled using the 
Aptina DevWare Software Package.

Macroscope characterization. The macroscope's optical performance (resolution and contrast) was determined by quantifying the device's limit of resolution (LOR) (Fig. 1), point spread function (PSF) (Suppl. Fig. 1), and modulation transfer function (MTF) (Suppl. Fig. 2) before any software-based enhancements. The LOR (spatial frequency limit) was defined by the bar

400 set where at least $5 \%$ contrast can be observed (the minimal contrast needed for detection by

401 the human eye) and measured with the "Find Peaks" ImageJ plugin and custom ImageJ scripts.

402 For PSF measurements, a point source was generated using a $1 \mu \mathrm{m}$ pinhole (TC-RT01,

403 Technologie Manufaktur), green fluorescence reference slide (2273, Ted Pella), and blue LED

404 excitation source (M470L3, Thorlabs). The lateral and axial FWHM of the measured PSF was

405 determined using Gaussian curve fits. The slight difference in our lateral LOR and PSF values is 406 likely due to the practical limitations in measuring the PSF, which is the convolution of the optics'

407 impulse responses (note that the LOR test does not allow axial resolution measurements). To

408 measure the modulation transfer function (MTF), we used the slanted edge test. Corresponding

409 data analysis was performed using the "Slanted Edge MTF" ImageJ plugin, Excel, and

410 GraphPad Prism. To quantify our device's contrast limit, we chose the MTF10 metric (i.e.,

411 contrast at $10 \%)$. Although MTF is frequently reported at $0 \%$, in our experience, MTF values

412 below $10 \%$ are affected by sensor noise limits and optical phase reversal. Note that the slanted

413 edge test allows for accurate measurements at spatial frequencies up to twice the Nyquist limit.

414 Our MTF10 results with line widths less than the size of a single pixel ( $3 \mu \mathrm{m}$ at the object plane)

415 are well above measurement error. Line widths are equal to one-half the spatial period.

418 To characterize the macroscope's FOV, we used a precision differential actuator (DRV3,

419 Thorlabs) to image a grid target printed directly on the surface of a microscope slide (500 $\mu \mathrm{m}$ 
420 grid spacing) (Fig. 1d). While our current illumination module was designed to provide a flat

421 illumination field, we observed moderate vignetting (i.e., image brightness reduction at the FOV

422 edges) (Fig. 1f). Apart from the vignetting common to large FOV imaging systems, this effect

423 resulted from a field stop we deliberately introduced for improving image quality at the FOV

424 edges. To compensate for this synthetic vignetting and enhance the signal-to-noise ratio (SNR),

425 an illumination module with a non-flat illumination field will need to be designed. This non-trivial

426 engineering task may involve using multiple optical elements, non-imaging type optics, or

427 miniature LEDs directly attached to the underside of the macroscope or baseplate. Currently,

428 we correct for vignetting with post-acquisition software. While this introduces digital noise (due

429 to the effectively reduced sensor bit depth at the FOV edges), this noise was not prominent in

430 our in vivo recordings because of the large $\Delta \mathrm{F} / \mathrm{F}$ signals we typically observed in live animals

431 (Fig. 2d-f).

432

433

The macroscope's working distance was measured by placing the device in contact with a grid target (R1L3S3P, Thorlabs) and then translating it upwards until the image came into focus, using a precision differential actuator (DRV3, Thorlabs). Note that the working distance depends on the cover glass thickness. We used a $\# 0$ cover glass ( 100 $\mu \mathrm{m}$ thick) for our experiments.

439 Tissue analogs were used to estimate attainable imaging depth (Suppl. Fig. 3). These tissue

440 phantoms consisted of $0.5 \%$ (by weight) agarose, $11 \%$ (by volume) scattering bead solution

441 (1.00 $\mu \mathrm{m}$ Polybead Microspheres; part no. 07310-15; Polysciences), and 5\% (by volume)

442 fluorescent bead solution (6 $\mu \mathrm{m}$ FocalCheck Microspheres; part no. F14807; ThermoFisher),

443 prepared using deionized water. Contrast and FWHM in Suppl. Fig. 3g-j were measured

444 without background subtraction using the ImageJ plugin MetroloJ. 
446 The integrated device weight was determined by weighing the individual components and the

447 assembled system. We did not include the sensor cabling wires or optical fiber in the

448 measurement as the weight of these is typically supported by external mounts or a commutator.

449

450 Experimental model and subject details. All procedures were performed following the

451 National Institutes of Health $(\mathrm{NIH})$ guidelines and were approved by the Institutional Animal

452 Care and Use Committee (IACUC) at the Salk Institute. Mouse strains used in this study

453 included GFAP-Cre (RRID: IMSR_JAX:012886), Ai95(RCL-GCaMP6f)-D (RRID:

454 IMSR_JAX:024105), and CX3cr1 $1^{+/ \text {eGFP }}$ mice (RRID: IMSR_JAX:005582). Mice were group-

455 housed, provided with bedding and nesting material, and maintained on a 12-h light-dark cycle

456 in a temperature $\left(22 \pm 1^{\circ} \mathrm{C}\right)$ and humidity controlled $(45-65 \%)$ environment. All the imaging and

457 behavioral experiments involved 7-9 weeks-old heterozygous female mice. Experimental mice

458 used in individual experiments typically originated from different litters. Mice had marks for

459 unique identification. No criteria were applied to allocate mice to experimental groups.

461 Live animal preparation. Animals were implanted with a spinal and head plate under general

462 anesthesia approximately one week before laminectomy, as previously described ${ }^{10}$. Buprenex

$463 \mathrm{SR}(0.5 \mathrm{mg} / \mathrm{kg})$ was given to minimize post-operative pain.

465 A laminectomy (typically 2 wide $x 4 \mathrm{~mm}$ long) was performed at the T12-T13 vertebra level,

466 corresponding to spinal segments $\mathrm{L} 2-\mathrm{L} 5^{4}$, four to seven days before the initial recording

467 session. The dura mater overlying the spinal cord was kept intact, and a custom-cut \#0

468 coverslip was used to seal the laminectomy creating an optical window for imaging. The

469 coverslip was replaced immediately before recording sessions to maximize optical clarity. 


\section{$471 \quad$ Fluorescence imaging}

472 In vivo imaging through a dorsal optical window. Data in behaving mice were acquired following

473 previously established protocols ${ }^{10}$. A fiber-coupled $473 \mathrm{~nm}$ DPSS laser was used for GCaMP6f

474 excitation. Approximately 10-20 recordings were taken per imaging session, with each recording

475 lasting around 1-2 min. Recordings were acquired at $\sim 25-75 \mu \mathrm{m}$ focal depth below the pia. The

476 typical average power for a given recording was $<105 \mu \mathrm{W} \mathrm{mm}{ }^{-2}$. No signs of phototoxicity, such

477 as a gradual increase in baseline fluorescence, lasting changes in activity rate, or blebbing of

478 labeled cells, were apparent in our recordings. All in vivo data were acquired at the image

479 sensor's full resolution (1,280 x 960 pixels) and maximum frame rate $(45 \mathrm{~Hz})$.

481 In vitro imaging through implanted microprisms. To demonstrate the wearable macroscopes'

482 long working distance, we implanted $2.0 \mathrm{~mm} \times 2.0 \mathrm{~mm} \times 2.0 \mathrm{~mm}$ microprisms $(\mathrm{W} \times \mathrm{D} \times \mathrm{H})$ in

483 tissue phantoms with embedded $6 \mu$ m-diameter fluorescent beads (Suppl. Fig. 3). The

484 miniature macroscope's FOV allowed imaging of the entire microprism face. Recordings were

485 taken at different focal depths from the vertical microprism-tissue phantom interface, where 0

$486 \mu \mathrm{m}$ was defined as the point when fluorescent beads first came into focus. A fiber-coupled 473

487 nm DPSS laser was used for fluorescence excitation.

488

$489 \quad$ Widefield imaging of tissue sections. To benchmark our wearable macroscope's imaging

490 performance, we used a commercial benchtop macroscope with similar FOV (Fig. 1e-f). This

491 macroscope was equipped with a wide-FOV objective (XL Fluor4x/340; Olympus Corp.) and a

492 high-resolution image sensor (ORCA FLASH4.0 V3, Hamamatsu Photonics K.K.). Despite being

493 orders of magnitude smaller and lighter, our wearable macroscopes provided comparable

494 resolution and contrast and $\sim 2 x$ better light collection due to their higher NA of 0.39 compared

495 to the commercial system's 0.28 . 


\section{Sensory stimuli and behavioral tests}

498 Sensory stimuli during in vivo imaging. Each imaging session consisted of up to 20 recordings.

499 Mechanical stimuli were delivered to the animal's tail using a rodent pincher system (cat. no.

500 2450; IITC Life Science, Inc.). Pinch pressures were applied in the dorsoventral direction at

501 approximately $6 \mathrm{~mm}$ from the base of the animal's tail. Each pinch stimulus (typically one per

502 recording) lasted around 1-2 s. Subsequent stimuli were delivered at least 1.5-2 minutes apart

503 to minimize response adaptation. The order in which stimuli of different amplitudes were

504 delivered was randomized. Fig. 2 shows data from $p<200 \mathrm{~g}$ and $\mathrm{p}>500 \mathrm{~g}$ mechanical stimuli.

Open-field test. To quantify how the $\sim 9.8 \mathrm{~g}$ macroscope mounted on the animal's back (lumbar

507 spinal cord) might affect general locomotor activity, we used the open-field test with infrared

508 tracking (ENV-510; Med Associates Inc.) (Suppl. Fig. 4). Animals ( 8-weeks-old female GFAP-

509 GCaMP6f mice with an implanted spinal plate) were acclimated to the testing environment by

510 placing their home cage in the testing room $\sim 60$ minutes before the recordings. Mice were then

511 transferred to individual testing chambers, always placed in the lower-left corner facing the

512 center of the apparatus. Six five-minute recordings were collected for each animal $(\mathrm{N}=3)$ with

513 the experimenter outside the room. The mice were then removed from the apparatus, the

514 wearable macroscope mounted, and placed back in their respective chambers using the same

515 starting orientation. Another six five-minute recordings were acquired with the experimenter

516 outside the room. Average running speed $(\mathrm{cm} / \mathrm{s})$, total distance traveled $(\mathrm{cm})$, and rearing

517 activity (vertical movement count) were analyzed. Recordings of a given animal were averaged

518 before averaging across animals.

521 In vivo imaging data. We used a custom Matlab script to convert the image sensor's raw files 
522 from 12- to 16-bit and adjust their pixel range. The data were then further processed in Fiji. We

523 converted the image data to TIFF, cropped the time-lapse recordings to the laser-on period and

524 fluorescently labeled central areas of the FOV, followed by computational illumination correction

525 and background subtraction. Full-frame image motion was reduced using Moco $^{19}$. Image edge

526 artifacts introduced by motion correction were cropped. Within-frame distortions were typically

527 small at the employed sampling rate $(45 \mathrm{~Hz})$ and therefore not corrected. Recordings for which

528 motion correction failed (e.g., vigorous running of $>100 \mathrm{~mm} / \mathrm{s}$ ) were excluded from data

529 analysis.

531 Motion-corrected calcium imaging data were analyzed using custom ImageJ and MATLAB

532 software. We used an unbiased tiling approach to quantify calcium activity across the FOV,

533 similar to our previous work ${ }^{20}$ (Fig. 2). Each tile corresponded to a $4 \times 4$-pixel (equivalent to $\sim 10$

$534 \times 10 \mu \mathrm{m}) \mathrm{ROI}$. We defined three ROI classes: over, near, and distant from blood vessels. First,

535 we calculated the mean and s.d. of the recording's maximum intensity image. Next, we

536 determined individual ROIs' average intensity values (A). If $A<$ mean - 1 s.d., it was considered

537 a blood vessel-ROI. If $A \geq$ mean $+(1+x)^{*}$ s.d. (with $x \in[0-1.2]$ depending on data set (e.g., blood

538 vessel size/pattern)), it was classified as distant from blood vessels. ROls with in-between

539 values were considered near-blood vessel-ROls. ROls over and near blood vessels were

540 excluded from data analysis. For all ROls distant from blood vessels, we calculated their

541 average fluorescence intensity over time. Corresponding activity traces were temporally

542 smoothed using an $0.4 \mathrm{~s}$ sliding average. Matlab's "findpeaks" function identified local maxima

543 within the traces. The fluorescence baseline and noise level (in s.d.) of a given trace were

544 calculated using a 2 s period before stimulus onset. Local maxima were considered evoked

545 activity if fluorescence intensity values surrounding the peak were at least 6 s.d. above baseline

546 for $\geq 2 \mathrm{~s}$. Calcium transient onset was defined as the point at which the fluorescence intensity 
547 trace immediately before the peak crossed a 2 s.d. threshold above baseline. Calcium transient

548 duration was defined as the trace's full width at half maximum (FWHM). Traces that showed a)

549 activity within $2 \mathrm{~s}$ after recording onset (i.e., before stimulus onset), b) fluorescence decreases

550 faster than the indicator's unbinding kinetics ( $\geq 50 \%$ signal drop within $\leq 250 \mathrm{~ms}$ ), or c) a 6 s.d.

551 drop below fluorescence baseline after transient onset for $\geq 3 \mathrm{~s}$ were considered artifactual (e.g.,

552 caused by tissue motion) and excluded from further analysis. For multi-peak transients, the

553 largest peak's value was used for FWHM calculation. If the intensity values between peaks fell

554 below the half-maximum value for $\geq 250 \mathrm{~ms}$, the half-maximum point closest to the largest peak

555 was used for FWHM calculation. If the trace's amplitude fell below 2 s.d. above baseline for

$556 \geq 400 \mathrm{~ms}$, the corresponding transients were considered separate calcium spikes.

To distinguish spontaneous from evoked calcium activity, we applied additional criteria. A

559 calcium transient was considered evoked if its onset occurred within $5 \mathrm{~s}$ after stimulus onset.

560 For all activity traces that passed the filters mentioned above, we calculated $\Delta \mathrm{F}(\mathrm{t}) / \mathrm{F}_{0}(\mathrm{t}) . \mathrm{F}_{0}$ was

561 determined with Matlab's mode function using a 15-bin width. Traces that showed abrupt

562 intensity changes ( $\geq 0.5 \Delta \mathrm{F} / \mathrm{F}$ within $\leq 65 \mathrm{~ms})$ were removed from the analysis.

564 To quantify potential differences in sensory-evoked activity on the left and right sides of the

565 spinal cord (Fig. 2j, k), we focused our analysis on $100 \mu \mathrm{m}$-wide gray matter regions $( \pm 150-250$

$566 \mu \mathrm{m}$ distance from the central vein).

568 Analog and video data processing and analysis. All analog data were synchronously

569 recorded at $1 \mathrm{kHz}$ using DAQExpress 2.0 software (National Instruments). Analog data included

570 the pressure sensor output from the rodent pincher system, treadmill speed, and on-off TTL

571 signal of the miniaturized macroscope's light source, depending on the experiment. Pinch 
572 application and mouse behavior were also recorded on a video camera ( $\geq 20 \mathrm{~Hz}$; Stingray F-

573 033, Allied Vision Technologies). To synchronize imaging with video data, we placed a near-

574 infrared LED within the video camera's FOV, triggered from the wearable macroscope's light

575 source drive signal (TTL pulse). Imaging and analog data were synchronized by recording the

576 on-off TTL signal of the macroscope's light source together with all other analog data. To relate

577 the animal's locomotor activity more closely to the imaging data, we focally restrained animals

578 on a spherical treadmill equipped with an optical encoder (E7PD-720-118, US Digital), allowing

579 precise readout of running speed. All analog data was processed using custom MATLAB

580 (Mathworks) routines. The pincher and encoder traces were first cropped to the light source-on

581 period. Pressure traces were then quantified with respect to stimulus amplitude and duration.

582 Encoder traces were smoothed using a sliding average (window size: $0.4 \mathrm{~s}$ ). Locomotion onset

583 or offset was defined as the point at which the smoothed running speed exceeded or fell below

$58410 \mathrm{~mm} / \mathrm{s}$. Encoder traces were analyzed concerning running speed, duration, and frequency. If

585 the running speed fell below the $10 \mathrm{~mm} / \mathrm{s}$ threshold for $\geq 750 \mathrm{~ms}$, the local maxima were

586 considered separate running bouts. The video data were cropped to the LED-on period. Videos

587 were scored manually regarding pinch onset and offset. Calcium transient latency was

588 calculated based on these measurements because video recordings provided higher temporal

589 resolution than the pincher traces. For population analysis, data was computationally sorted

590 using custom MATLAB (Mathworks) routines. Only trials with $1.5 \pm 0.5 \mathrm{~s}$ pinch duration were

591 included in the analysis.

592

\section{Statistical analysis}

594 All data were analyzed and plotted using MATLAB, Excel, or GraphPad Prism software. Paired 595 t-tests were used to evaluate potential calcium activity differences on the left and right sides of 596 the spinal cord (Fig. 2j, k) and changes in the animal's general locomotor activity (Suppl. Fig. 
597 4). All data are represented as mean \pm s.e.m. Group sample sizes were chosen based on power

598 analysis or previous studies. The following convention was used to indicate $P$ values: 'NS'

599 indicates $P>0.05$, ‘*' indicates $0.01<P \leq 0.05$, ‘**' indicates $0.001<P \leq 0.01$, ‘***' indicates

$600 \quad 0.0001<P \leq 0.001$, and '****' indicates $P \leq 0.0001$.

601

602 Reporting summary

603 Further information on research design is available in the Research Reporting Summary linked

604 to this paper.

605

606

Data availability

607 The data that support the findings of this study will be deposited in the Brain Image Library (BIL;

608 https://www.brainimagelibrary.org/index.html). They will also be available from the

609 corresponding author upon reasonable request.

610

611 Code availability

612 The custom Java- and Matlab-based code used to process and analyze the data will be

613 deposited in GitHub. It will also be available from the corresponding author upon reasonable

614 request.

615

616 
bioRxiv preprint doi: https://doi.org/10.1101/2021.12.23.474042; this version posted December 24,2021 . The copyright holder for this preprint

(which was not certified by peer review) is the author/funder, who has granted bioRxiv a license to display the preprint in perpetuity. It is made available under aCC-BY-NC-ND 4.0 International license.

\section{References}

61819 Dubbs, A., Guevara, J. \& Yuste, R. moco: Fast Motion Correction for Calcium Imaging.

619 Front Neuroinform 10, 6, doi:10.3389/fninf.2016.00006 (2016).

62020 Patriarchi, T. et al. Ultrafast neuronal imaging of dopamine dynamics with designed

621 genetically encoded sensors. Science 360, doi:10.1126/science.aat4422 (2018). 


\section{Acknowledgments}

623 We thank members of the Nimmerjahn lab for comments on the manuscript, the Salk machine

624 shop and Nick Andrews of the Behavior Testing Core for technical support, and J. Chambers for

625 mouse colony management. This work was primarily supported by the National Institutes of

626 Health (NIH) grant R01NS108034 (A.N). It was partially supported by the NIH grants

627 U01NS103522, U19NS112959, U19NS123719, a Salk Innovation Grant, and equipment funds

628 from C. and L. Greenfield (A.N.). P.S. was supported by a Rose Hills Foundation graduate

629 fellowship and N.A.N. by funds from an NIH T32/CMG Training Grant, Burt and Ethel Aginsky

630 Research Scholar Award, Kavli-Helinski Endowment Graduate Fellowship, and NIH individual

631 predoctoral fellowship (F31NS120619). The content is solely the authors' responsibility and

632 does not necessarily represent the official views of the $\mathrm{NIH}$.

\section{Author contributions}

634 P.S., D.D., and A.N. conceived and designed the study with input from E.M.C. and N.A.N. P.S.

635 developed and characterized the wearable macroscopes and wrote ImageJ-based data analysis

636 code. D.D. performed the in vivo imaging experiments. E.M.C. conducted the motor behavior

637 experiments. P.S., D.D., and E.M.C. analyzed the in vitro and in vivo data. A. Ngo, G.G., and

638 J.A.O. developed Matlab- and ImageJ-based data analysis code. A.N. supervised the study and

639 wrote the initial manuscript draft. All authors contributed to the text and figures, discussed the

640 results, or provided input and edits on the manuscript.

\section{Competing interests}

642 The authors declare no competing interests.

643

644 Correspondence and requests for materials should be addressed to A.N. 


\section{Supplementary Figures}
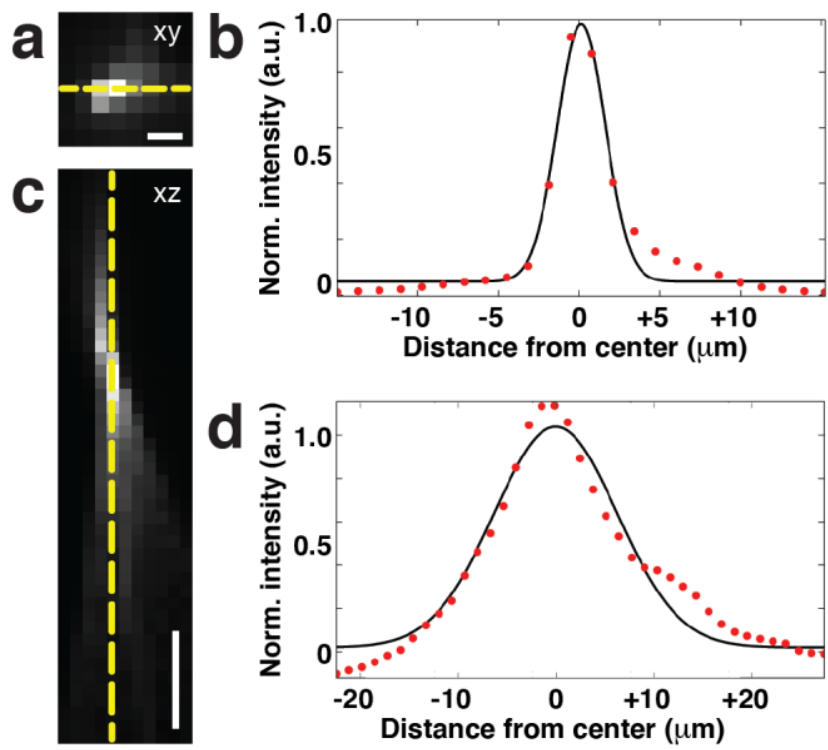

647 Suppl. Fig. 1 | Wearable macroscopes with custom-compound microlenses provide $~ 3$

$648 \mu \mathrm{m}$ lateral resolution. a, Experimentally measured lateral point spread function (PSF) of the

649 integrated wearable macroscope. Scale bar, $5 \mu \mathrm{m} . \mathbf{b}, \mathrm{x}$ cross-section showing a full width at half

650 maximum (FWHM) of $\sim 3.3 \mu \mathrm{m}$. c, Experimentally measured axial PSF of the integrated

651 wearable macroscope. Scale bar, $20 \mu \mathrm{m}$. b, z cross-section showing an FWHM of $\sim 17.7 \mu \mathrm{m}$.

652 The red dotted and black solid lines in $b$ and $d$ show the measured intensity (along the yellow

653 dashed lines in a and c) and Gaussian fit profile, respectively. 
a MTF (FOV center)

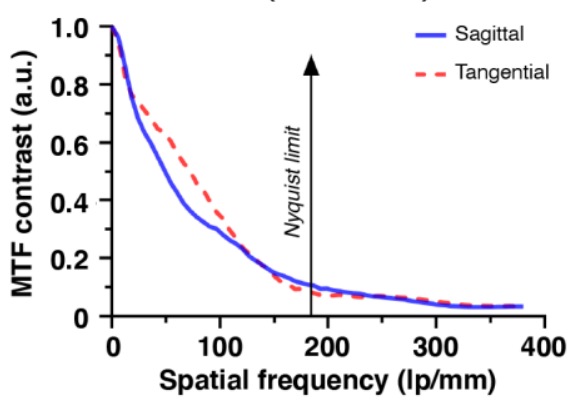

b MTF (mid-left-804 $\mu \mathrm{m})$

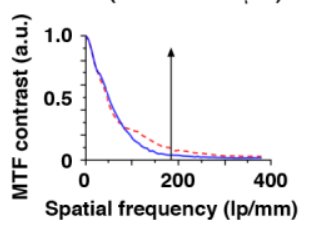

MTF (mid-right/+804 $\mu \mathrm{m})$
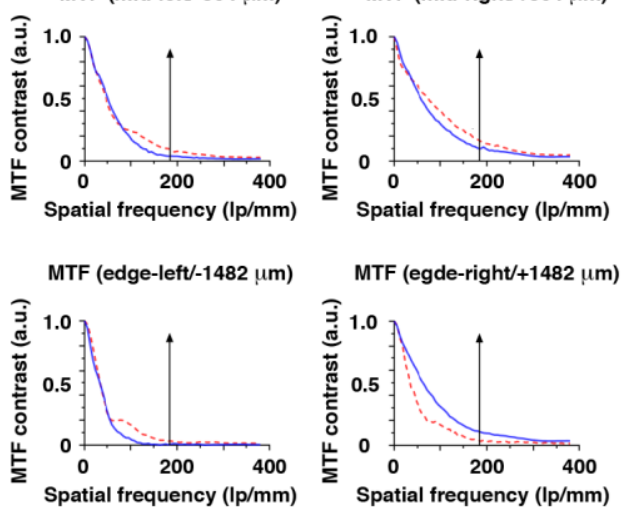

C

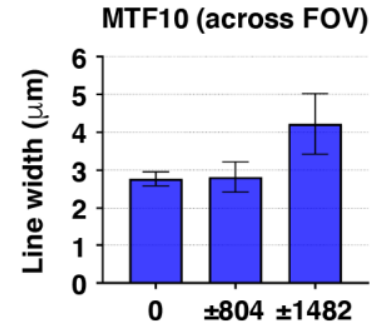

Distance from FOV center $(\mu \mathrm{m})$

658 Suppl. Fig. 2 | Wearable macroscopes with custom-compound microlenses provide high

659 contrast across the field of view. a, Modulation transfer function (MTF) of the integrated

660 macroscope measured in the center of the field of view (FOV) using the Slanted Edge test. b,

661 MTF at different indicated FOV positions relative to the center. c, MTF contrast at 10\% (MTF10)

662 across the FOV. Displayed values are averages across similar FOV locations and horizontal

663 and vertical Slanted Edge targets. Spatial frequencies were converted to line widths. The data

664 are presented as mean \pm s.e.m. The larger error bars toward the FOV edge likely indicate

665 sample tilt. 

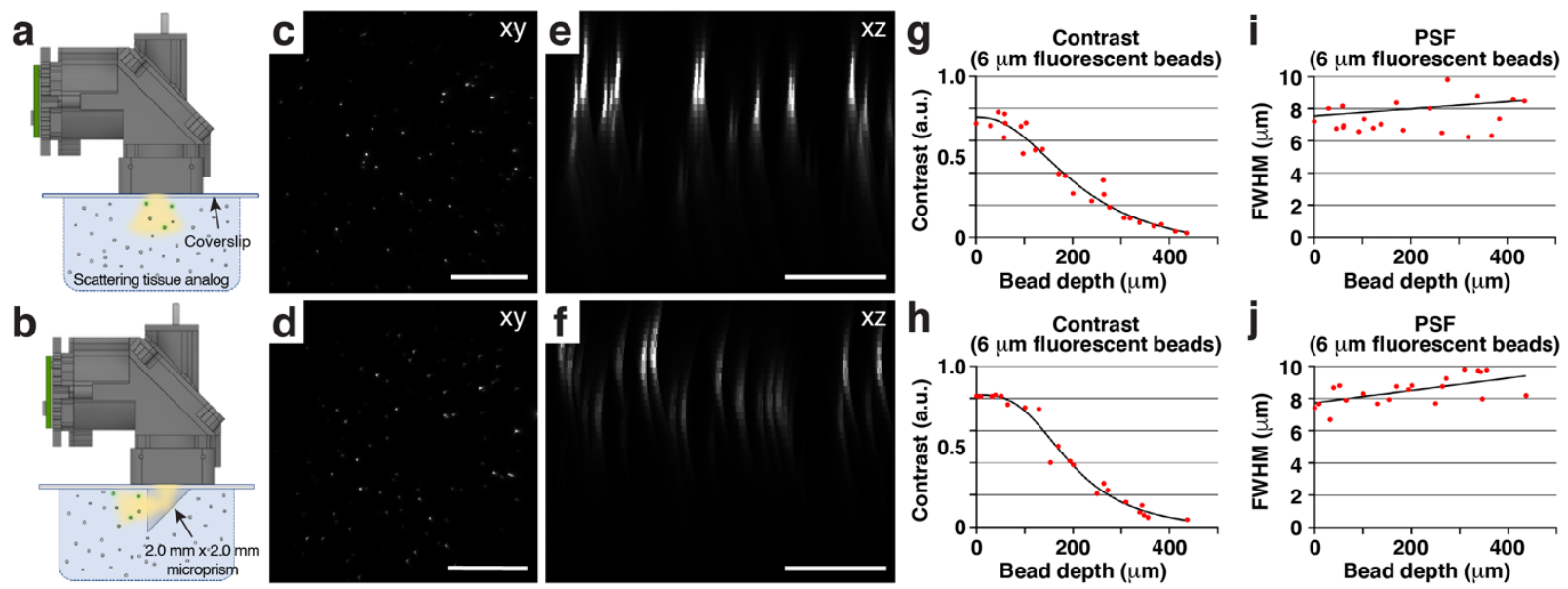

Suppl. Fig. 3 | Wearable macroscopes with custom-compound microlenses permit

670 imaging through implanted microprisms. a-b, Schematics showing the experimental

671 approach for characterizing and comparing two imaging conditions in scattering tissue

672 phantoms (Methods): Top, imaging through a coverslip; bottom, imaging through a coverslip

673 with an attached $2.0 \mathrm{~mm} \times 2.0 \mathrm{~mm} \times 2.0 \mathrm{~mm}$ microprism $(\mathrm{W} \times \mathrm{D} \times \mathrm{H})$. c-d, Example images of

674 tissue phantom embedded $6 \mu \mathrm{m}$-diameter fluorescent beads. Each image is a maximum

675 intensity projection through a z-stack acquired as shown in a-b by translating the wearable

676 macroscope axially. Scale bar, $500 \mu \mathrm{m}$. e-f, Maximum intensity side projections of the acquired

677 z-stacks. Scale bar, $200 \mu \mathrm{m} . \mathbf{g}-\mathbf{h}$, Bead contrast as a function of imaging depth. i-j, Lateral full

678 width at half maximum (FWHM) of the $6 \mu \mathrm{m}$-diameter fluorescent beads as a function of imaging

679 depth. 
a

Open field test

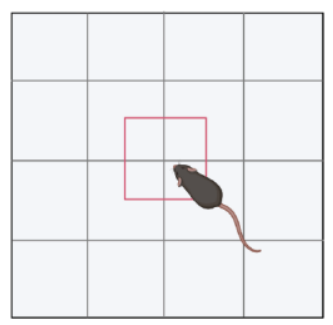

C

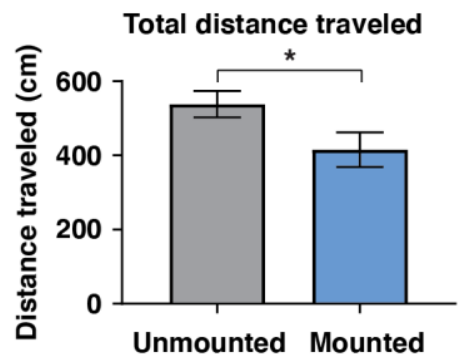

b

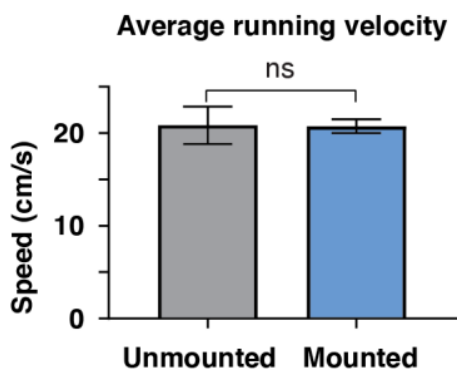

d

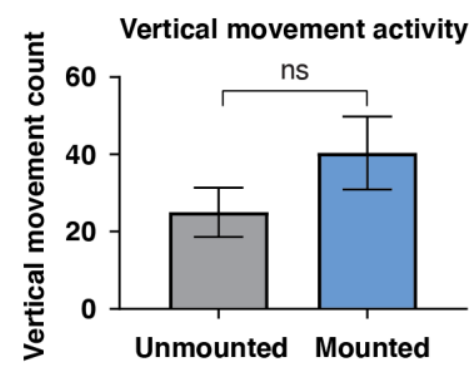

Suppl. Fig. 4 | Spine-mounted macroscopes of $<10$ grams have little effect on mouse

open-field behavior. a, Schematic of the open-field test used to compare the animal's general locomotor activity with or without the $\sim 9.8 \mathrm{~g}$ macroscope mounted over its lumbar spinal cord. b-

687 data in b-d are from six five-minute recordings before and after attaching the device. All animals

$688(\mathrm{~N}=3)$ were tested before and after macroscope mounting (Methods). Paired t-tests determined 
691 Supplementary Tables

\begin{tabular}{|c|c|c|c|c|c|}
\hline Surface & Type & Radius & Thickness & Glass & Diameter \\
\hline OBJ & STANDARD & Infinity & 0.40000 & N-BK7 & 6.00000 \\
\hline 1 & STANDARD & Infinity & 3.83988 & & 5.95166 \\
\hline 2 & STANDARD & -5.74334 & 1.60002 & N-SF66 & 5.36800 \\
\hline 3 & STANDARD & 15.70964 & 0.81873 & & 6.41800 \\
\hline 4 & STANDARD & -32.74580 & 1.60104 & S-LAH97 & 6.89400 \\
\hline 5 & STANDARD & -8.05357 & 0.21700 & & 7.61200 \\
\hline 6 & STANDARD & Infinity & 1.60035 & N-LAF34 & 8.46000 \\
\hline 7 & STANDARD & -12.51519 & 0.52205 & & 8.77400 \\
\hline 8 & STANDARD & 21.51710 & 1.60150 & S-LAH55V & 9.00000 \\
\hline 9 & STANDARD & -47.46657 & 0.50000 & & 9.00000 \\
\hline 10 & STANDARD & Infinity & 1.00000 & FSILICA & 8.92000 \\
\hline 11 & STANDARD & Infinity & 5.50000 & & 8.81600 \\
\hline 12 & COORDBRK & - & 0.00000 & & - \\
\hline 13 & STANDARD & Infinity & 0.00000 & MIRROR & 12.21416 \\
\hline 14 & COORDBRK & - & 5.50000 & & - \\
\hline STO & STANDARD & -33.34728 & 1.98940 & S-LAH97 & 7.12400 \\
\hline 16 & STANDARD & 6.13755 & 1.00000 & N-SF66 & 7.23800 \\
\hline 17 & STANDARD & 17.95736 & 0.10000 & & 7.75400 \\
\hline 18 & STANDARD & -6.35500 & 1.89487 & S-LAH58 & 8.06200 \\
\hline 19 & STANDARD & -35.88850 & 1.60925 & & 7.63800 \\
\hline 20 & STANDARD & Infinity & 0.74100 & & 6.07952 \\
\hline 21 & STANDARD & 15.94777 & 0.99962 & N-SF66 & 5.53400 \\
\hline 22 & STANDARD & -21.55705 & 2.80317 & & 5.09800 \\
\hline 23 & STANDARD & Infinity & 0.10000 & N-BK7 & 4.08338 \\
\hline IMA & STANDARD & Infinity & & & 4.05844 \\
\hline
\end{tabular}




\section{Supplementary Movies}

\section{Suppl. Movie 1 | High-speed trans-segmental imaging of innocuous tail pinch-evoked}

697 calcium activity in behaving GFAP-GCaMP6f mice. Example time-lapse recording acquired

698 with the wearable macroscope showing innocuous tail pinch-evoked calcium excitation in spinal

699 astrocytes. The data were obtained at $\sim 45 \mathrm{fps}$ and $\sim 50 \mu \mathrm{m}$ focal depth below the pia. Elapsed

700 time is indicated in the upper right corner (total duration: $35 \mathrm{~s}$ ). To allow the precise readout of

701 locomotor activity, the mouse was placed on a spherical treadmill. Innocuous pinch triggered

702 sparse calcium excitation across the imaged lumbar spinal segments. Running alone did not

703 evoke significant calcium increases. Scale bar, $250 \mu \mathrm{m}$.

704

705 Suppl. Movie 2 | High-speed trans-segmental imaging of noxious tail pinch-evoked

706 calcium activity in behaving GFAP-GCaMP6f mice. Example time-lapse recording acquired

707 with the wearable macroscope showing noxious tail pinch-evoked calcium excitation in spinal

708 astrocytes. The data were obtained at $\sim 45 \mathrm{fps}$ and $\sim 50 \mu \mathrm{m}$ focal depth below the pia. Elapsed

709 time is indicated in the upper right corner (total duration: $34 \mathrm{~s}$ ). To allow the precise readout of

710 locomotor activity, the mouse was placed on a spherical treadmill. Noxious pinch triggered

711 widespread, coordinated calcium excitation on both sides of the spinal cord and across lumbar

712 spinal segments. Scale bar, $250 \mu \mathrm{m}$. 